\title{
Functional intron-derived miRNAs and host-gene expression in plants
}

\author{
Umidjon Shapulatov ${ }^{1,2}$, Mark van Hoogdalem', Marielle Schreuder ${ }^{1}$, Harro Bouwmeester ${ }^{1}$, \\ Ibrokhim Y. Abdurakhmonov ${ }^{2}$ and Alexander R. van der Krol ${ }^{1 *}$ (1)
}

\begin{abstract}
Background: Recently, putative pre-miRNAs locations have been identified in the introns of plant genes, raising the question whether such genes can show a dual functionality by having both correct maturation of the host gene premRNA and maturation of the miRNAs from the intron. Here, we demonstrated that such dual functionality is indeed possible, using as host gene the firefly luciferase gene with intron (ffgLUC), and different artificial intronic miRNAs (aimiRNA) placed within the intron of ffgLUC.

Results: The miRNAs were based on the structure of the natural miR319a. Luciferase (LUC) activity in planta was used to evaluate a correct splicing of the ffgLUC mRNA. Different target sequences were inserted into the aimiRNA to monitor efficiency of silencing of different target mRNAs. After adjusting the insertion cloning strategy, the ffgLUCaimiR-319a gene showed dual functionality with correct splicing of ffgLUC and efficient silencing of TEOSINTE BRANCHED1/ CYCLOIDEA/PROLIFERATING CELL FACTOR1 transcription factor genes targeted in-trans by aimiR-319a or targeting the transgene ffLUC in-cis by an aimiR-LUC. Silencing of endogenous target genes by aimiRNA or amiRNA is efficient both in transient assays and stable transformants. A behave as strong phenotype the PHYTOCHROME B (PHYB) gene was also targeted by ffgLUC aimiR-PHYB. The lack of silencing of the PHYB target was most likely due to an insensitive target site within the PHYB mRNA which can potentially form a double stranded stem structure.
\end{abstract}

Conclusion: The combination of an overexpression construct with an artificial intronic microRNA allows for a simultaneous dual function in plants. The concept therefore adds new options to engineering of plant traits that require multiple gene manipulations.

Keywords: Intron, imiRNA, aimiRNA, miRNA, amiRNA, luciferase

\section{Background}

Important traits of crop plants have successfully been manipulated by selection of mutants [1], by ectopic expression of a transgene [2-4], or by silencing of a single gene $[5,6]$. However, because of the complexity of gene-networks in plants, the effect of many single-gene disturbances is limited due to buffering capacity of such networks [7, 8]. Moreover, plant trait manipulation may potentially benefit from synergistic interaction between independent transgene manipulations. Stacking of

\footnotetext{
*Correspondence: Sander.vanderKrol@wur.nl

${ }^{1}$ Laboratory of Plant Physiology, Wageningen University,

Droevendaalsesteeg 1, 6708 PD Wageningen, The Netherlands

Full list of author information is available at the end of the article
}

independent transgenes is time-consuming, especially in crops that are difficult targets for transformation. Engineering in recalcitrant crops may therefore benefit from techniques that can target multiple genes by a single transformation event.

MicroRNAs (miRNA) are short (19-22nt) non coding RNAs that can silence the expression of specific target genes and natural miRNAs form an integral part of developmental decisions in plants $[9,10]$. From all plant miRNAs listed in the microRNA database (http://www. mirbase.org/) only a small number have been functionally characterised. Moreover, while most miRNA are processed from regular non-coding miRNA-genes, recently, protein-coding genes with introns containing potential miRNA sequences have been identified both in mammals 
and plants. For instance, the Arabidopsis thaliana genome contains 37 protein coding genes with intronic miRNAs (imiRNAs) and the rice genome contains 181 protein coding genes with imiRNAs [11]. At present, there is no experimental evidence that plant genes containing imiRNAs show simultaneous dual functionality: a correct intron splicing of the host gene pre-mRNA to form a mRNA encoding a functional protein and processing of the miRNA from the intron for effective silencing of the target gene. For instance, in some cases the miRNA encoded in the intron is only produced as alternatively spliced transcript [12]. In such cases, correct mRNA splicing and gene expression and miRNA production from the intron may be mutually exclusive. Functionality of intron-derived miRNAs has been demonstrated in mammals, C. elegans, zebra fish, and chicken [13]. It has been demonstrated that an imiRNA can be correctly processed from the intron sequence, without interfering with the accuracy of the splicing process of the host gene $[14,15]$. The intron-derived miRNAs require type-II RNA polymerases (Pol-II) and spliceosome components for their biogenesis. In animals, it has been shown that regular miRNA processing is dependent on Droshamediated cleavage, but initial processing of some imiRNAs are Drosha-independent. Instead, initial imiRNA processing is coupled to the intron splicing reaction [16]. How imiRNAs are processed in plants is not fully known at present. Introns and active $5^{\prime}$ splice sites $\left(5^{\prime} \mathrm{ss}\right)$ have been shown to stimulate the accumulation of miRNAs encoded within the first exons of intron-containing MIR genes and Knop et al. found that the $5^{\prime}$-splice site is crucial for the regulation of intronic miRNA-402 biogenesis from the first intron of host gene At1g77230 [17]. Moreover, the gene encoding dicer protein DCL1 contains imiR838 in intron 14 and the gene can produce both functional DCL1 mRNA and mature miR838. In this instance the imiRNA biogenesis and DCL1 mRNA biogenesis are mutually exclusive but in a population both gene products may be produced [18].

Here, we tested whether a protein coding transgene can be effectively expressed in plants, while also producing a functional miRNA. The feasibility and requirements for such dual gene functionality were determined using three gene construct (ffgLUC ${ }^{\text {aimiR-319a, }}$,fgLUC ${ }^{\text {aimiR-LUC }}$ and ffgLUC ${ }^{\text {aimiR-PHYB }}$ ), designed to report on both protein and miRNA function. For overexpression, the firefly Luciferase gene with a single intron (ffgLUC) was used, which allows for easy monitoring of gene activity and splicing accuracy. As template for the miRNA sequence in the intron, the sequence of the natural ath-miR319a was used [19]. For alternative targets, the 21-bp sequence targeting TEOSINTE BRANCHED1/CYCLOIDEA/PROLIFERATING CELL FACTOR1 (TCP) transcription factor in
miR319a was replaced by a 21 nucleotide sequence targeting ffgLUC mRNA [20] or 21-bp targeting the Arabidopsis thaliana PHYB mRNA (AT2G18790). Initially, insertion of the miRNA into the LUC intron resulted in a loss of LUC activity, indicating incorrect splicing of the intron from the LUC pre-mRNA. However, after adjusting the miRNA position within the intron, the transgene showed normal LUC activity when expressed in plants, indicating accurate splicing of the LUC pre-mRNA. Moreover, the aimiRNA targeting TCPs or ffgLUC both were able to suppress target gene expression, indicating effective processing of the aimiRNA from the ffgLUC intron. The concept of a transgene containing an aimiRNA could be useful for simultaneous manipulation of several gene activities, which could be an important tool for plant biotechnology.

\section{Methods}

\section{Plant materials and growth condition}

Arabidopsis thaliana (Col-0 background, N1092) was used for stable transformation. The Arabidopsis phyB-9 T-DNA insertion mutant (\#CS6217) was obtained from the NASC stock collection. Plants were grown on rockwool in a growth chamber at $12 \mathrm{hL} / 12 \mathrm{hD}$ at $22{ }^{\circ} \mathrm{C}$ on half strength Hoagland-nutrient solution.

\section{Cloning of expression constructs}

Artificial microRNAs constructs were created using ath-miR319a backbone as described by Liang [20]. The primer sequences used are listed in Additional file 1: Table S2. The artificial miRNA nucleotide sequences 5'-TAACTGTAAACCGAAAGGCTG- $3^{\prime}$ for the Ath$P H Y B$ (AT2G18790) were selected using WMD3-Web MicroRNA Designer (http://wmd3.weigelworld.org/ cgi-bin/webapp.cgi). The IDT RNAi design tools (Integrated DNA Technologies) was used to design the amiRNA nucleotide sequence targeting the luciferase mRNA (5'-TAGAACTGCCTGCGTCAGATT-3'). PremicroRNA 319a was amplified directly from $A$. thaliana genomic DNA using primers (CAAACACACGCTCGG ACGCAT-F and CATGGCGATGCCTTAAATAAAGR). The aimiRNA sequences were amplified from premiRNA319a using specific primers which added EcoR $\mathrm{V}$ and EcoR I restriction sites for cloning into the intron of ffgLUC (GATATCAGAGAGCTTCCTTGAGTCCAT TCAC-F and GAATTCAGGGAGCTCCCTTCAGTC CAATC-R). For amplification of the aimiR-LUC the TCP target sequence in the primers was replaced by the selected LUC target sequence (GATATCTATAACTGC CTGCCTCAGATAAGGTCGTGATATGATTCA-F and GAATTCTAGAACTGCCTGCGTCAGATTAAAGAG AATCAATGATCCA-R). For the amplification of the aimiR-PHYB the TCP target sequence in the primers was 
replaced by the selected PHYB target sequence (GAT ATCTAGCTGTAAACCGTAAGGCTCAGGTCGTGA TATGATTCA-F and GAATTCTAACTGTAAACCGAA AGGCTGAAAGAGAATCAATGATCCA-R). To generate of ffgLUC ${ }_{\text {del }}^{\text {aimiR319a }}$ construct the first exon plus 10 nucleotides from $5^{\prime}$ site of intron was amplified by using primer which introduce Nco I at start codon and EcoRV site in intron (CCATGGAAGACGCCAAAAAC-F and GATATCAGAAACTTACGTAATGTTCACCTCG-R). The second exon plus 61 base pair from $3^{\prime}$ site of the intron was amplified using primers which introduce an EcoR I site at the end of the intron sequence and an Not I site after the stop codon (GAATTCAACTTTTCTAAT ATATGACCAAAATTTGTT-F and GCGGCCGCTTA CAATTTGGACTTTCCGCCCTT-R). To generate of ffgLUC $^{\text {imiR319a }}$ ffgLUC $^{\text {aimiR-LUC }}$ and ffgLUC ${ }^{\text {aimiR-PHYB }}$ constructs, the first exon plus 33 nucleotides from the $5^{\prime}$ end of the intron was amplified by using primer pairs introducing an Nco I at the ATG start codon and an EcoR V site at the end of the intron sequence (CCATGGAAG ACGCCAAAAAC-F and GATATCTACTAATTAATG ATAATTATT-R). The second exon of ffgLUC was amplified from 135 base pairs from $3^{\prime}$ splice site to after the stop codon, introducing an EcoR I site at in the intron and Not I site after the stop codon, using the primer pairs (GAATTCGTAATATAATATTTCAAATATTTTTTT CAAAATAA-F and GCGGCCGCTTACAATTTGGA CTTTCCGCCCTT-R). The resulting PCR products were digested with EcoR I and EcoR V and the amiRNAs product was ligated into the ffgLUC intron. The ffgLUC was amplified with primers introducing an Nco I site at the ATG and Not I site after the stop codon (CCATGGAAG ACGCCAAAAAC-F and CGGCCGCTTACAATTT GGACTTTCCGCCCTT-R). The ffgLUC, ffgLUC ${ }^{\text {imiR319a, }}$ ffgLUC $^{\text {aimiR-LUC }}$ or ffgLUC ${ }^{\text {aimiR-PHYB }}$ constructs were subsequently ligated into the Nco I/Not I sites of pIVA2.1 entry vector which contained double $35 \mathrm{~S}$ promoter and RubescoS terminator. To generate the binary vector, all pIVA2.1-based vectors were cloned into the pKGW_RedSeed vector [21] through gateway based site-specific recombination technology with one way LR reaction. The pKGW RedSeed vector contains a DsRed marker gene that is expressed in the seed coat which allows for selection of T0 transformed seeds.

For confirmation of LUC or PHYB silencing in trans the artificial microRNAs $2 \times 35 \mathrm{~S}::$ amiR-LUC and $2 \times 35$ S::amiR-PHYB constructs were generated using primer sets which replace the TCP target sequence in miR-319a with target sequences for LUC or PHYB respectively (LUC: CCATGGTATAACTGCCTGCCT CAGATAAGGTCGTGATATGATTCA-F and GCGGCC GCTAGAACTGCCTGCGTCAGATTAAAGAGAAT CAATGATCCA-R or PHYB: CCATGGTAGCTGTAA
ACCGTAAGGCTCAGGTCGTGATATGATTCA-F and GCGGCCGCTAACTGTAAACCGAAAGGCTGAAA GAGAATCAATGATCCA-R). The PCR products were cloned into pIVA2.1 entry vector which was subsequently used for recombination into the $\mathrm{pKGW}$ _RedSeed vector. All destination vectors were subsequently transformed to Agrobacterium tumefaciens (AGL0).

\section{Plant transformation and selection transformants}

Agrobacterium tumefaciens was used for plant transformation using the floral dip method as described [22]. Transgenic $\mathrm{T}_{0}$ seeds were identified by DsRed pigmentation of the seed coat. For germination seeds were plated on 3\% water agar plates and cold-treated for 5 days at $4{ }^{\circ} \mathrm{C}$ after which plates were incubated in growth chambers in the light at room temperature. After 3 days, germinated seedlings were transferred to soil or rock wool for plant growth.

\section{Agrobacterium-mediated transient expression in N.benthamiana leaves}

Agro-infiltration in $N$. benthamiana using agrobacterium strains carrying the different expression vectors (or empty vector) was done as described by Wang [23].

\section{LUC activity measurement}

For LUC activity measurements in stable transformed Arabidopsis thaliana plants were sprayed with $1 \mathrm{mM}$ D-luciferin (Duchefa, Haarlem, NL) $24 \mathrm{~h}$ and $1 \mathrm{~h}$ before imaging with an $\left(-80{ }^{\circ} \mathrm{C}\right)$ air-cooled CCD Pixis 1024B camera system (Princeton Instruments, Massachusetts, USA) equipped with a $35 \mathrm{~mm}, 1: 1.4$ Nikkon SLR camera lens (Nikon, Tokyo, Japan) fitted with a DT Green filter ring (Image Optics Components Ltd, Orsay, France) to block chlorophyll fluorescence. Exposure time is as indicated.

For transient assays, N.benthamiana leaves were harvested 4 days post agro-infiltration. Leaves were sprayed with $1 \mathrm{mM}$ D-luciferin at 24 and $1 \mathrm{~h}$ before imaging (5 min exposure time). Relative luminescence from LUC activity was analysed in Image J (Bethesda, Maryland, USA), using background subtraction. For each treatment LUC activity in leaves from 6 to 8 independent plants was quantified.

\section{Hypocotyl length measurement}

For hypocotyl length measurement, seeds were surface sterilized and imbibed on $0.25 \%$ water agar plates at $4{ }^{\circ} \mathrm{C}$ in the dark, after which plates were transferred to a Red LED light box (50 uMol) at $22{ }^{\circ} \mathrm{C}$. Seedlings were flattened at 5 days after transfer, and hypocotyl length was determined from photograph in Image J (Bethesda, 
Maryland, USA). At least 20 seedlings were scored from each genotype.

\section{Quantitative RT-PCR}

For RNA analysis, $T_{3}$ generation plants were grown for 4 weeks. The RNA was extracted from rosette leaves from WT (Col-0), ffgLUC $C_{\text {del }}^{\text {aimiR-319a }}$, ffgLUC $C^{\text {aimiR-319a }}$ or ffgLUC ${ }^{\text {aimiR-PHYB }}$ transformants using InviTrap Spin Plant RNA mini Kit (Berlin, Germany), following manufacturer's instructions. Purified total RNAs were subjected to TURBO DNA-free ${ }^{\mathrm{TM}}$ DNase (Thermo Fisher Scientific Inc., Waltham, Massachusetts) treatment to avoid with contaminated genomic DNA. For reverse transcription the iScrip II mix reagent was used that included $10 \mathrm{mM}$ oligo (dT) primer according to the manufacturer's instruction (Bio-Rad, CA,USA). The primers listed in Additional file 1: Table S2 were used for the real time qPCR. Reaction were carried out with RNA isolated from pooled samples from three individual plants, with triple biological replicates using SYBR Green PCR Master Mix (Bio-Rad, CA,USA) on the CFX Connect Real Time System machine (Bio-Rad, CA, USA). For Arabidopsis the $A$. thaliana ACTIN1 was used as reference. RNA analysis from transient assays in $N$. benthamiana were carried out on RNA isolated from three pooled agroinfiltrated leaves, in triple biological replicates, using N.benthamiana UBI3 as reference genes. The Ct method $(2-\Delta \Delta \mathrm{Ct})$ was used to analysis the differences in mRNA values (http://www.bio-rad.com/). All expression constructs used in the transient assays contain a 35S::DsRed marker gene and quantification of the DsRED gene expression in the transient assays was used to confirm similar transformation frequencies in the different agroinfiltration treatments.

\section{Small RNA extraction and stem-loop RT-PCR assays}

Detection of specific small RNAs was by the step-loop PCR method as described by Varkonyi-Gasic [24]. Briefly, leaf material was collected from ffgLUC (as control), ffgLUC $C_{\text {del }}^{\text {aimiR-319a }}$, ffgLUC ${ }^{\text {aimiR-319a, and ffgLUC }}{ }^{\text {aimiR-PHYB }}$ plants and immediately ground in liquid nitrogen with a mortar and pestle. Approximately $100 \mathrm{mg}$ ground leaf tissue was used to small RNA extraction. The extraction of small RNAs were performed by using Prima microRNA Isolation Kit (Lot\#SLBL6958 V, Sigma Aldrich, USA) according to the manufacturer's protocol. The small RNA purity and concentration was measured by NanoDrop spectrophotometer (Thermo Scientific, USA).

The specific RT primers were used for miR319a and amiR-PHYB in stem-loop RT reaction. Reverse transcription reaction were performed according to VarkonyiGasic et al. [24]. Forward primers for mature miR319a or amiR-PHYB and universal reverse primer (see Additional file 1: Table S2) were used in RT-PCR. The PCR amplification products analysed by gel-electrophoresis on a $4 \%$ agarose gel in 1xTAE buffer.

\section{Statistical analyses}

Comparison of means was analysed for statistical significance with a 2 -sample $t$ test $(P<0.001)$.

\section{Results}

ffgLUC gene with intron-deletion miR-319a (ffgLUC $C_{\text {del }}^{\text {aimiR-319a }}$ ) shows only single functionality: impaired LUC $m$ RNA splicing but efficient silencing of TCP targets

To determine whether a functional microRNA can be efficiently generated from an intron of a transgene, without affecting accuracy of intron splicing, both accuracy and efficiency of transgene splicing and efficiency of silencing by the aimiRNA need to be monitored. To monitor transgene splicing the firefly luciferase (ffgLUC) reporter gene with an intron was used [25] (see Fig. 1). To study the efficiency of target gene silencing, the precursor of the native miRNA319a, which targets several members of the Arabidopsis TCP transcription factor family, was used [26]. When the artificial intron-miRNA, aimiR-319a, is correctly processed, it should be active and elicit a leaf growth phenotype similar to that induced by $2 \times 35 \mathrm{~S}:: \mathrm{miR}-319 \mathrm{a}$ [20].

The initial cloning procedure for insertion of the miR-319a precursor sequence into the intron of ffgLUC resulted in a 37 base pair deletion in the ffgLUC intron (for sequence see Additional file 1: Fig. S1). This gene is named ffgLUC $C_{\text {del }}^{\text {aimiR-319a }}$ (see Fig. 1, 2). In ffgLUC $C_{\text {del }}^{\text {aimiR-319a }}$ the intron branch point and both $5^{\prime}$ and $3^{\prime}$ intron border sequences remained intact (see Additional file 1: Fig. $\mathrm{S} 1)$. The ffgLUC $C_{\mathrm{del}}^{\text {aimiR-319a }}$ was cloned into a binary expression vector under control of the enhanced CaMV $2 \times 35 \mathrm{~S}$ promoter and a red seed coat transformation marker gene [21]. The ffgLUC $C_{\mathrm{del}}^{\mathrm{aim} i \mathrm{R}-319 \mathrm{a}}$ expression construct was introduced into Agrobacterium tumefaciens and activity of the constructs was tested both by transient expression in N.benthamiana leaves and by stable transformation of A.thaliana. In the transient expression assay in $N$. benthamiana, LUC activity of ffgLUC $C_{\text {del }}^{\text {aimiR-319a }}$ was compared to that of a ffgLUC at 4 days post-agro infiltration. Results show a high LUC activity in leaves expressing ffgLUC, but only low LUC activity for leaves expressing ffgLUC $C_{\text {del }}^{\text {aimiR-319a }}$ (Fig. 2a). This indicates that intron splicing accuracy from ffgLUC $C_{\mathrm{del}}^{\text {aimiR-319a }}$ is impaired compared to that of ffgLUC. The transient expression assays in $N$. benthamiana are not suitable to assess if aimiR-319a elicits a leaf phenotype. Therefore, we tested whether endogenous $N$. benthamiana TCP4 (NbTCP4) gene expression was affected by ffgLUC $C_{\text {del }}^{\text {aimiR-319a }}$ as the AthTCP target sequence of aimiR-319a shows substantial overlap with 


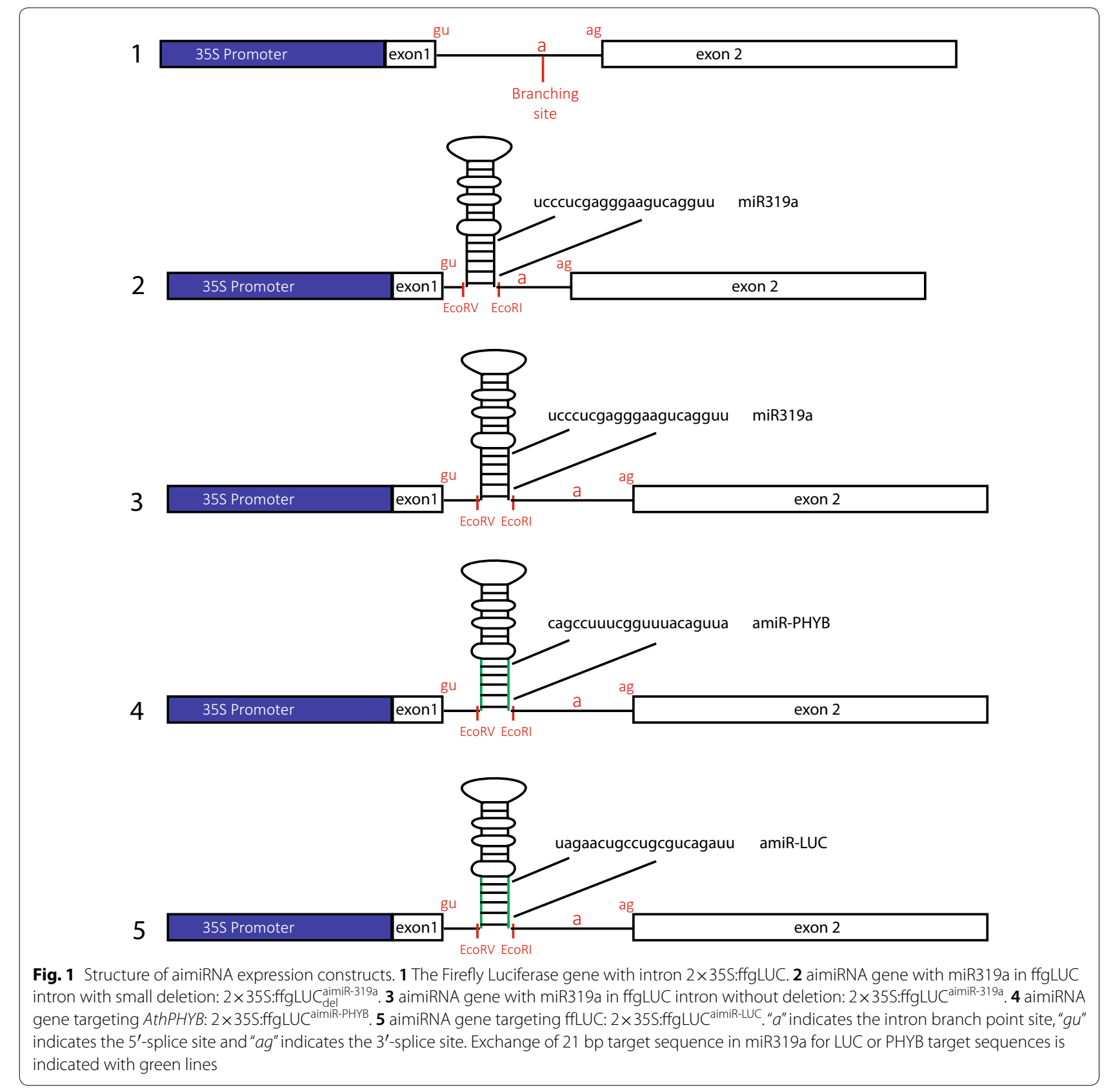

sequences in NbTCP4. Results show that NbTCP4 expression level was reduced in leaves expressing ffgLUC $C_{\text {del }}^{\text {aimiR- }}$ 319a compared to the control leaves expressing ffgLUC (Fig. 2a).

Transformants of Arabidopsis thaliana with the ffgLUC del dimi-319a or ffgLUC expression constructs were identified in $\mathrm{T}_{0}$ seeds by expression of the red seed coat marker present in the binary vector (Additional file 1: Table S1). From the red ffgLUC del $^{\text {aimiR-319a }} \mathrm{T}_{0}$ seeds 19 independent transformants were grown. All these plants showed a leaf growth phenotype (data not showed) as described for plants expressing $2 \times 35 \mathrm{~S}:: \operatorname{miR} 319 \mathrm{a}$ [20], indicating that the miR319a is efficiently processed from aimiR-319a in stable transformed plants. Indeed, expression analysis of the miR319a target genes AthTCP2, AthTCP3, AthTCP4 indicated that their expression was reduced by $\sim 90 \%$ in the ffgLUC $C_{\text {del }}^{\text {aimiR-319a }}$ plants (Fig. 2b). However, the LUC activity in plants expressing ffgLUC ${ }_{\text {del }}^{\text {aimiR-319a }}$ is low compared to control plants (expressing ffgLUC) of the same age (Fig. 2c). Both the 
(See figure on next page.)

Fig. 2 Single activity of ffgLUC Cel aimir-319a in transient assays and stable transformed plants. a Left: LUC activity of ffgLUC and ffgLUC del $C_{\text {del }}$-319a at 4 days post-agro-infiltration in Nicotiana benthamiana transient assay. Significant differences between samples $\left(^{* * *}\right.$ ) is based on standard error (student's $t$ test, $P<0.01$ ). Right: RT-PCR analysis of NbTCP4 expression in transient assay with ffgLUC or ffgLUC aimiR-319a. Quantification of the DsRED gene expression was used to confirm similar transformation efficiencies in the agro-infiltration with ffgLUC and ffgLUC CaimiR-319a (Additional file 1: Fig. S3). b Left: Representative stable transformed Arabidopsis rosette plant and leaf expressing ff-gLUC or ffgLUC del aimi-319a. Right: (reference gene AthActin 1, expression of TCP 2, 3 and 4 each normalized to that in one WT plant). Significant differences between samples (***) is based on standard error (student's $t$ test, $P<0.01$ ). c LUC activity in representative stable transformant Arabidopsis expressing ff-gLUC or ffgLUC Cel $C_{\text {de }}$-319a . Graph: quantified LUC expression of eight independent transformants expressing either ff-gLUC or ffgLUC Cel aimiR-319a . $\mathbf{d}$ Top: PCR forward and reverse primer positions

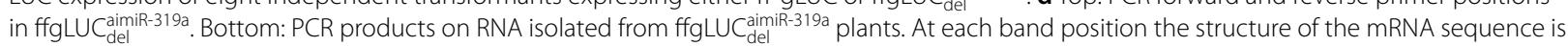
shown (see also Additional file 1: Fig. S2)

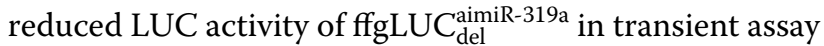
and stable transformants suggest an incorrect maturation of the luciferase pre-mRNA derived from the ffgLUC $\mathrm{del}_{\mathrm{del}}^{\mathrm{am}}$ 319a. Indeed, PCR analysis of the luciferase mRNA across the intron splice site showed that there were multiple aberrant products and only very low levels of correctly spiced luciferase mRNA derived from ffgLUC $C_{\text {del }}^{\text {aim-319a }}$ (Fig. 2d). Presumably, the dual action at the intron in luciferase pre-mRNA by both an intron-splicing proteincomplex and an miRNA processing protein-complex leads to spatial interference, which in this case especially affects correct maturation of the pre-mRNA. Sequence analysis of the aberrant PCR products showed that both aberrant $3^{\prime}$ - and $5^{\prime}$ splice site selection occurred, while the major PCR product was derived from unspliced mRNA (Fig. 2d and Additional file 1: Fig. S2). To solve the putative spatial interference during processing of ffgLUC $C_{\text {del }}^{\text {aimiR-319a }}$ mRNA maturation, we next adapted the cloning strategy for miRNA insertion into the intron.

\section{ffgLUC aimiR-319a displays dual functionality: correct LUC mRNA splicing and TCP silencing}

The miRNA insertion cloning strategy was adapted by direct insertion of the aimiRNA into the ffgLUC intron, without deletion of intron sequence, resulting in the expression construct ffgLUC ${ }^{\text {aimiR-319a }}$ (Fig. 1 and Additional file 1: Fig. S1). The ffgLUC ${ }^{\text {aimiR-319a }}$ expression construct was introduced into Agrobacterium tumefaciens and was again tested both by transient expression in $N$. benthamiana and by stable transformation of Arabidopsis. In the transient expression assays, the activity of ffgLUC ${ }^{\text {aimiR-319a }}$ was compared with that of ffgLUC without intronic miR319a (Fig. 3a). This resulted in a similar LUC activity in leaf tissue expressing either ffgLUC $^{\text {aimiR-319a }}$ or ffgLUC, suggesting an efficient and accurate splicing of the intron from ffgLUC ${ }^{\text {aimiR-319a }}$ mRNA (Fig. 3a). To test the functionality of aimiR-319a in targeting TCP genes in N. benthamiana, NbTCP4 mRNA level was checked by RT-PCR in control treatments and leaves expressing ffgLUC ${ }^{\text {maimiR-319a }}$. NbTCP4 expression was reduced by $60 \%$ in leaves infiltrated with
$\mathrm{ffgLUC}^{\text {aimiRNA319a }}$, suggesting that a functional miRNA319a can be produced from aimiR-319a (Fig. 3a).

From the stable transformation of Arabidopsis, $\mathrm{T}_{0}$ seeds expressing the red seed coat marker were selected, from which 19 independent T1 transformants were grown (Additional file 1: Table S1). Out of these 19 plants, two plants did not survive, while 17 plants produced $\mathrm{T}_{1}$ seeds. Each of these $17 \mathrm{~T}_{1}$ plants showed the phenotype associated with constitutively overexpressed native miR319a $[20,27]$ (Fig. 3b). This indicates that an miR319a was efficiently processed from imiR-319a located in the intron of ffgLUC ${ }^{\text {aimiR-319a }}$ in stably transformed plants, leading to efficient silencing of TCP genes. This is also confirmed by qPCR analysis of RNA isolated from a representative ffgLUC $^{\text {aimiR-319a }}$ transformant, which shows $>90 \%$ reduction in TCP2, TCP3 and TCP4 mRNA levels compared to plants expressing conventional ffgLUC (Fig. 3b). Nevertheless, LUC activity in the same ffgLUC ${ }^{\text {aimiR-319a }}$ transformant is similar compared to the ffgLUC control (Fig. 3c). These results indicate that the luciferase premRNA is correctly spliced and simultaneously aimiR319a provides silencing of TCPs in transformed plants.

\section{aimiR-LUC silences ffgLUC in-cis in stable transformants, but not in transient assays}

An aimiRNA was made targeting the luciferase mRNA itself (aimiR-LUC). The aimiR-LUC is based on the sequence and structure of the native miR319a precursor, but the 21 base-pair sequences targeting TCPs are replaced by 21 base-pairs targeting luciferase mRNA (Fig. 1 and Additional file 1: Fig. S1). In cells expressing


LUC transgene from which it is derived (silencing incis). Both mature aimiR-LUC and LUC mRNA are produced from the same pre-mRNA and silencing of LUC activity provides information on the relative efficiency of the two maturation processes (mRNA vs miRNA). In the transient expression assays, LUC activity in $N$. benthamiana leaves expressing ffgLUC ${ }^{\text {aimiR-LUC }}$ showed no significant reduction compared with leaves expressing ffgLUC (Fig. 4a). This indicates correct splicing of the luciferase pre-mRNA, but no effective silencing 


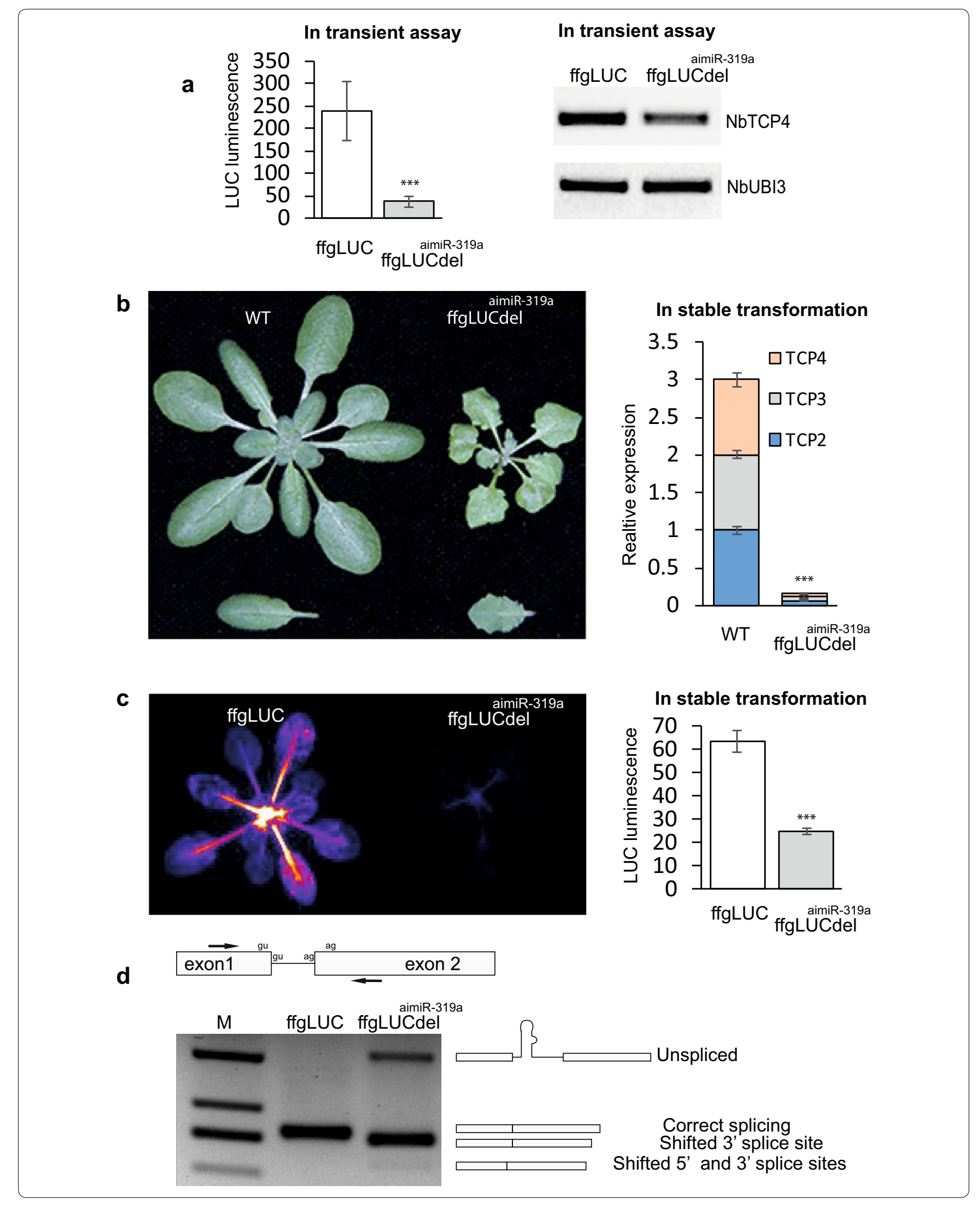


a

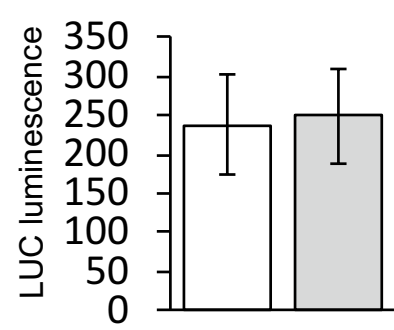

ffgLUC ffgLUC $^{\text {aimik-319a }}$

\section{In transient assay}



b

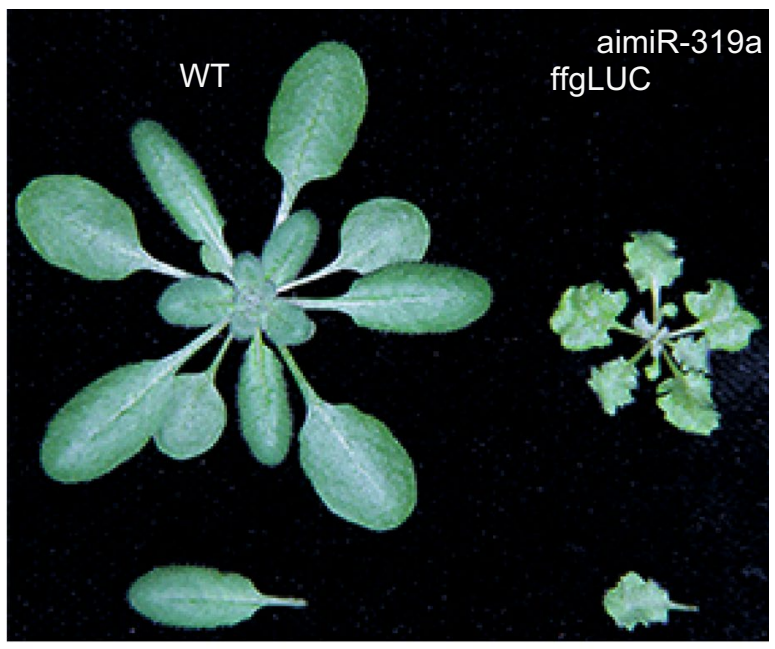

C

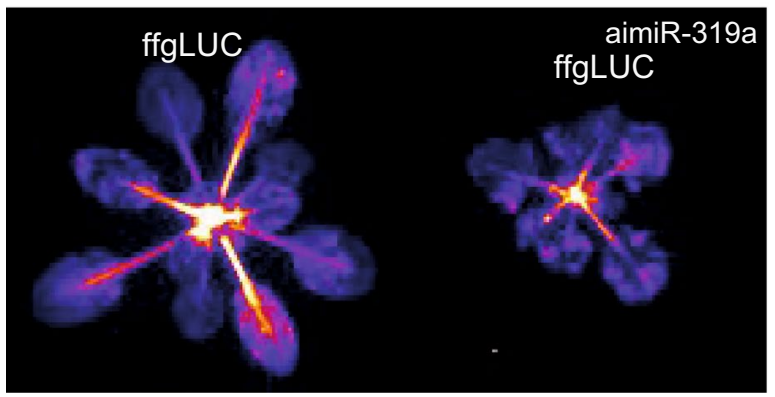

In stable transformation

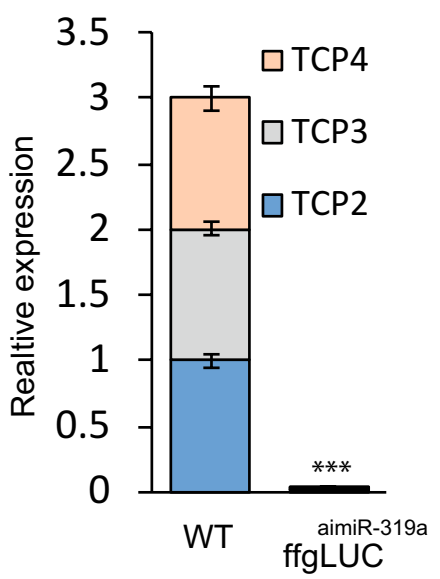

In stable transformation

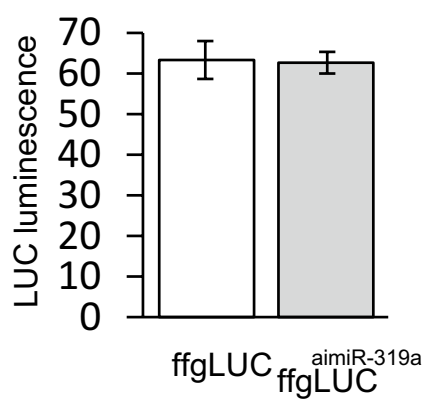

Fig. 3 Dual activity of ffgLUC aimiR-319a in transient assays (a) and stable transformed plants (b, c). a Left: LUC activity of ffgLUC and ffgLUC ${ }^{\text {aimiR-319a at }}$ 4 days post-agro-infiltration in Nicotiana benthamiana transient assay ( $n=$ five leaves per treatment). Right: RT-PCR analysis of NbTCP4 expression in transient assay with ffgLUC or ffgLUC Cel dimi-319a . Quantification of the DsRED gene expression was used to confirm similar transformation efficiencies in the agro-infiltration with ffgLUC and ffgLUC $C^{\text {aimiR-319a }}$ (Additional file 1: Fig. S3). b Left: Representative stable transformed Arabidopsis thaliana rosette plant and leaf expressing ff-gLUC or ffgLUC CaimiR-319a Right: average relative expression level of TCP2/3/4 genes in five WT and ffgLUC del aimi-319a plants (reference gene AthActin 1, expression of TCP2,3 and 4 each normalized to that in one WT plant). Significant differences between samples (***) is based on standard error (student's $t$ test, $P<0.01$ ). c Left: LUC activity in representative stable transformant Arabidopsis thaliana expressing ff-gLUC or ffgLUC ${ }^{\text {aimiR-319a }}$. Right: quantified LUC in expression of eight independent transformants expressing either ff-gLUC or ffgLUC ${ }^{\text {aimiR-319a }}$

by aimiR-LUC in-cis. To compare the silencing in-cis with silencing in-trans in the transient assay, an ffcLUC (LUC cDNA) expression construct was co-infiltrated with a $2 \times 35$ S::amiR-LUC expression construct. This showed that also $2 \times 35 \mathrm{~S}::$ amiR-LUC is not capable of silencing transiently expressed LUC (Fig. 4a). Combined, these results indicate that efficient maturation 


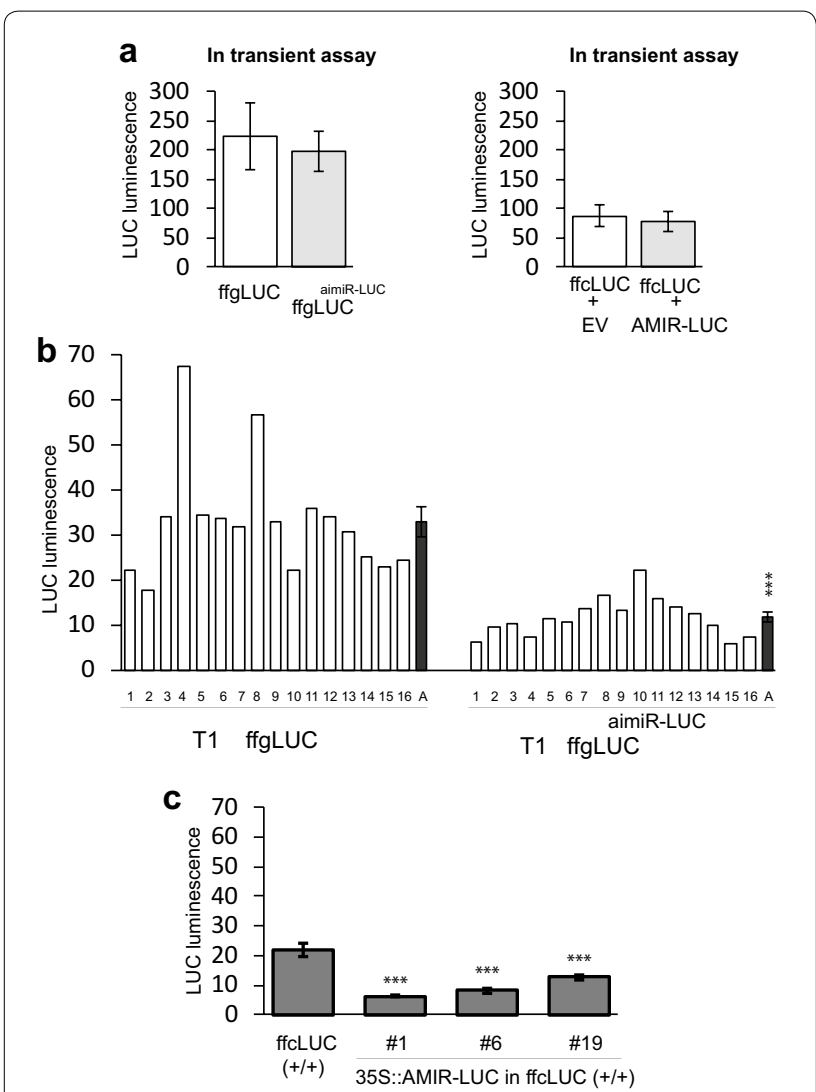

Fig. 4 Evaluation of silencing in cis- and trans in transient assays (a) and stable transformed plants (b, c). a Left: evaluation silencing in-cis transient assay: LUC activity of ffgLUC and ffgLUC ${ }^{\text {aimir-LUC }}$ at 4 days post-agro-infiltration in Nicotiana benthamiana transient assay ( $n=$ five leaves per treatment). Right: evaluation silencing in-trans transient assay: LUC activity in Nicotiana benthamiana transient assay of ffCLUC co-infiltrated with empty vector and ffCLUC co-infiltrated with amiR-LUC at 4 days post-agro-infiltration $(n=$ five leaves per treatment). Quantification of the DsRED gene expression was used to confirm similar transformation efficiencies in the agro-infiltration with ffgLUC and ffgLUC aimiR-LUC (Additional file 1: Fig. S3). b Evaluation of silencing in-cis: Relative LUC activity in sixteen independent $T_{1}$ generation of ffgLUC and ffgLUC CaimiR-LUC plants. "A" indicates average LUC activity in set of transgenic plants. Significant differences between samples $\left(^{* *}\right)$ is based on standard error (student's $t$ test, $P<0.01)$. c Evaluation of silencing in-trans: Relative LUC activity in homozygous ffcLUC line and T1 of same ffcLUC line transformed with $2 \times 35$ S:amiR-LUC. Significant differences between samples (***) is based on standard error (student's $t$ test, $P<0.01$ )

of luciferase mRNA from ffgLUC ${ }^{\text {aimiR-LUC }}$ occurs upon transient expression but that silencing by aimiR-LUC or amiR-LUC is not effective under these conditions.

The ffgLUC and ffgLUC ${ }^{\text {aimiR-LUC }}$ binary vectors were also stably transformed into Arabidopsis and $\mathrm{T}_{0}$ seeds with the red seed coat were identified (Additional file 1: Table S1). For each transformation event, 16 independent transformants were grown and LUC activity was quantified in independent transformed plants at 21 days post germination. On average, the LUC activity was reduced by $65 \%$ in the 16 individual ffgLUC aimiR-LUC $\mathrm{T}_{1}$ plants compared to that in 16 individual $\mathrm{T}_{1}$ ffgLUC plants (Fig. 4b). This indicates that amiR-LUC is efficiently processed from aimiR-LUC in stably transformed plants. For comparison of silencing in-cis with silencing in-trans in stably transformed plants, one line expressing $2 \times 35$ S::ffcLUC was transformed with a $2 \times 35 \mathrm{~S}$ ::amiRLUC expression construct. In three T2 double transformants (homozygous for both $2 \times 35$ S::ffcLUC and $2 \times 35$ S::amiR-LUC) the LUC activity was reduced by 69-53\% compared to the original ffcLUC line (Fig. 4c). Silencing efficiency in-trans therefore seems to be in the same range as silencing efficiency in-cis.

\section{ffgLUC aimiR-PHYB shows efficient mRNA maturation, but no silencing of AthPHYB}

In addition to the aimiRNA targeting TCP and LUC, an aimiRNA targeted against the PHYB mRNA of Arabidopsis thaliana was tested. The aimiR-PHYB was again placed at the same intron position as in the functional ffgLUC $^{\text {imiR-319a }}$ and ffgLUC ${ }^{\text {aimiR-LUC }}$ constructs. The aimiR-LUC is based again on the miR319a but with a replacement of the 21 base pairs in miR319a targeting TCP by 21 base pairs targeting AthPHYB mRNA (ffgLUC $^{\text {aimiR-PHYB }}$ ) (Fig. 1 and Additional file 1: Fig. S1). In transient assays, the leaf tissues expressing ffgLUC $^{\text {aimiR-PHYB }}$ showed similar LUC activity as leaves expressing ffgLUC (Fig. 5a), again indicating efficient and accurate maturation of the luciferase pre-mRNA from the ffgLUC ${ }^{\text {aimiR-PHYB }}$ expression construct.

After stable transformation of Arabidopsis thaliana with ffgLUC ${ }^{\text {aimiR-PHYB }}, \mathrm{T}_{0}$ seeds with the red seed coat were identified (Table 1) and 10 independent transformed $\mathrm{T}_{1}$ plants were grown. From these, eight transformants with a single copy transgene insertion were selected for further analysis. On average, the LUC activity in these eight lines was comparable with that of eight independent transformants expressing ffgLUC (Fig. 5b). This again indicates that also the intron in ffgLUC ${ }^{\text {aimiR-PHYB }}$ is efficiently and correctly spliced from the luciferase premRNA. In contrast, the silencing of PHYB is not effective in ffgLUC ${ }^{\text {aimiR-PHYB }}$ lines as expression of PHYB mRNA is similar in ffgLUC ${ }^{\text {aimiR-PHYB }}$ and ffgLUC lines (Fig. 5c). The silencing of PHYB mRNA expression can also be tested in a bioassay. When seedlings are grown under constant Red (cR) light, lines with reduced PHYB expression are expected so show enhanced hypocotyl elongation. Ten independent homozygous $\mathrm{T} 1$ lines of ffgLUC $\mathrm{C}^{\text {aimiR-PHYB }}$ were germinated under $\mathrm{cR}$. While the hypocotyl length of a phyB-9 mutant was elongated compared with WT, the hypocotyl length of the ten ffgLUC ${ }^{\text {aimiR-PHYB }}$ 
a
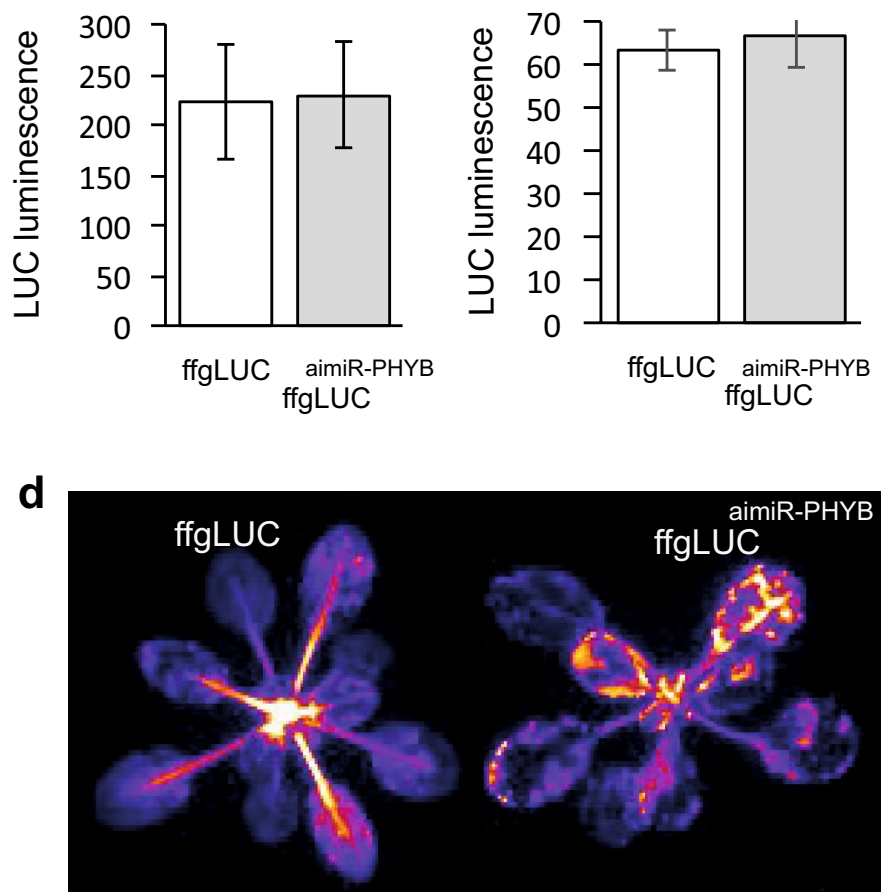

C In stable transformants
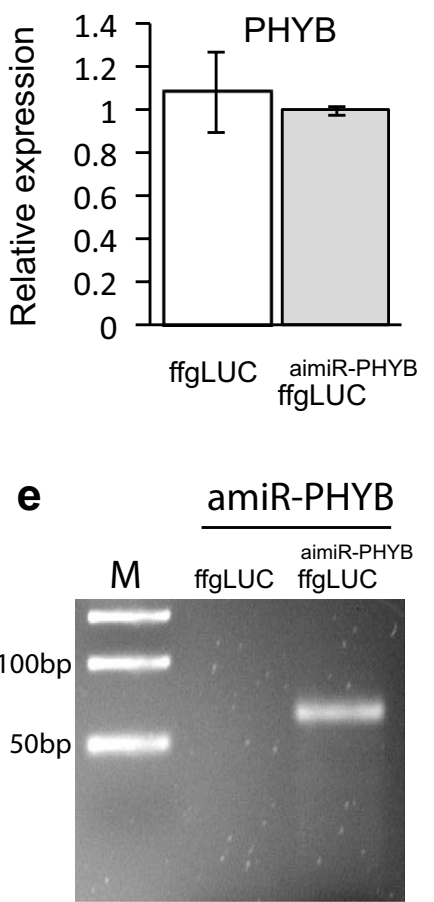

Fig. 5 Activity of ffgLUC ${ }^{\text {aimiR-PHYB }}$ in transient assays (a) and stable transformed plants (b, c). a Relative LUC activity of ffgLUC and ffgLUC aimiR-PHYB at 4 days post-agro-infiltration of Nicotiana benthamiana leaves ( $n=$ five leaves per treatment). $\mathbf{b}$ Relative LUC activity in eight independentstable transformants of Arabidopsis thaliana expressing either ff-gLUC or ffgLUC ${ }^{\text {aimiR-PHYB }}$. c Average relative expression level of AthPHYB in five ff-gLUC and ffgLUC CimiR-PHYB plants (reference gene AthActin 1, expression of AthPHYB normalized to that in one ffgLUC plant). $\mathbf{d}$ Image of LUC activity in representative transgenic plants expressing ff-gLUC (left) or ffgLUC ${ }^{\text {aimiR-PHYB }}$ (right). e Detection of mature amiR-PHYB by stem-loop RT PCR analysis in small RNA isolated from ffgLUC CaimiR-PHYB plants but not in small RNA isolated from control ffgLUC plants

transformants was not statistically different from WT (data not shown). All together, these results suggest that the mature amiR-PHYB derived from ffgLUC ${ }^{\text {aimiR-PHYB }}$ is not functional in silencing PHYB expression.

\section{Discussion}

\section{Functional aimiRNA requires sufficient spacing in intron}

Intron-derived miRNAs (imiRNAs) are an alternative source for miRNAs in mammals and plants [16, 2831]. Evidence has been obtained that functional miRNAs can be derived from imiRNAs in mammalian cells and plants $[32,33]$ but plant genes containing intronic miRNA sequences have only been studied sparsely. Here, we demonstrate that the concept of an imiRNA can be used to construct a transgene with dual functionality: overexpression of the transgene and silencing of an endogenous target gene of interest. Our constructs demonstrate that the structural sequence information of the pre-miRNA mi319a is sufficient for full functionality when placed correctly into an intron, allowing for both normal maturation of the pre-mRNA and for generation of a functional mature microRNA.

In all aimiRNA constructs tested here, the aimiRNA was inserted into an 189 long intron sequence of the ffgLUC gene. In the first construct the insertion was done at $10 \mathrm{bp}$ from the $5^{\prime}$-end of the LUC-intron sequence. For this construct the LUC activity was low compared to ffgLUC control construct in both in transient and stable (Fig. 2a, c). This indicates that a certain distance is needed between the $5^{\prime}$-splice site and the imiRNA insertion site for efficient pre-mRNA maturation. It could be that the reduced distance between $5^{\prime}$-splice site and imiRNA sequence in ffgLUC $C_{\text {del }}^{\text {aimiR-319a }}$ resulted in spatial constraints because of simultaneous assembly of spliceosome and miRNA-processing protein complexes. In contrast, the aimiRNA placed at $55 \mathrm{bp}$ from the $5^{\prime}$-splice site resulted in efficient maturation of the LUC mRNA, resulting in similar LUC activity for ffgLUC ${ }^{\text {aimiRNA-319a }}$ and ffgLUC in transient expression as well as stable transformants (Fig. 3a, c). It was not investigated whether mRNA and aimiRNA derive from the same pre-mRNA 


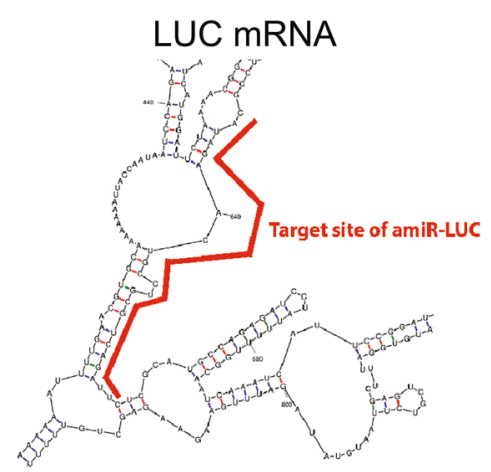

TCP2 mRNA

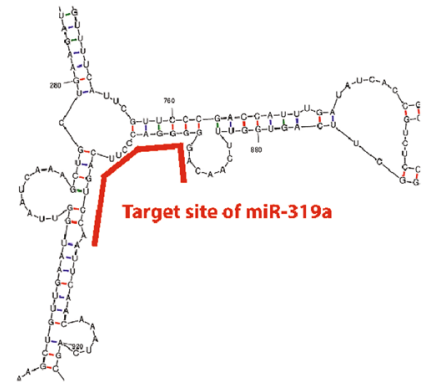

PHYB mRNA

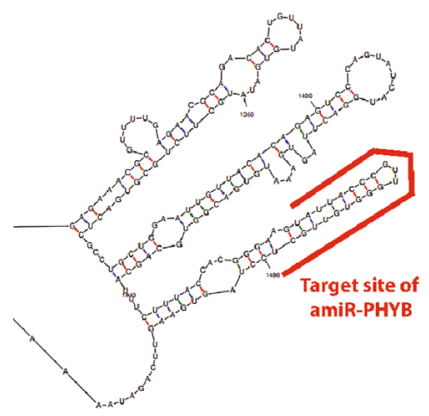

Fig. 6 Predicted secondary structure of mRNAs targeted by miRNA. RNA secondary structure prediction by UNAFold (http://unafold.rna.alban y.edu/) [37]

transcript or whether the two mature products are produced mutually exclusive. However, since LUC activity from ffgLUC ${ }^{\text {aimiRNA-319a }}$ is similar as from ffgLUC it suggests the same level of mRNA production from both constructs. If part of the pre-mRNA is exclusively used for mature amiRNA production and the other part for mature ffgLUC mRNA production we would expect a lower LUC activity from ffgLUC aimiRNA-319a, which is not the case (Fig. 3). Whether both products (mRNA, aimiRNA) are indeed derived from the same pre-mRNA needs further investigation but for practical purposes the ffgLUC ${ }^{\text {aimiRNA }}$ constructs seem to function as dual functional transgenes.

The imiRNA positioning within the intron may be further improved for functionality, for which positioning of natural imiRNA in plant genes may be used as a guide. The average length of introns is $101 \mathrm{bp}$ in Arabidopsis and $160 \mathrm{bp}$ in rice $[34,35]$. By contrast, the average length of introns containing imiRNAs is $625 \mathrm{bp}$ in Arabidopsis and $2178 \mathrm{bp}$ in rice [11]. Therefore, it may still be possible that a larger distance between inserted miRNA and the $5^{\prime}$ - and $3^{\prime}$ splice sites enhances functionality of the imiRNA (more efficient splicing and processing to miRNA).

\section{Efficiency of silencing is function of both aimiRNA and target gene expression level}

The construct ffgLUC ${ }^{\text {aimiRNA-LUC }}$ with the miRNA targeting the LUC mRNA in-cis showed $\sim 65 \%$ reduction in LUC activity, which is very similar to the silencing in-trans reached by a $2 \times 35 \mathrm{~S}$ ::amiR-LUC in stably transformed plants (Fig. 4b, c). Presumably the LUC mRNA and aimiR-LUC are produced in equal molar amounts from ffgLUC aimiRNA-LUC pre-mRNA, suggesting that miRNA needs to be in excess to its target mRNA in order to obtain higher levels of silencing. For instance, silencing of the TCP transcription factor genes, which are expressed at low levels, by the aimiRNA is very efficient (Fig. 2b, 3b). In contrast to the stable transformed plants, the constructs targeting LUC mRNA in-cis or in-trans are not effective in transient assays. In transient assays the gene copy number is artificially high and may result in saturation of the gene silencing machinery.

\section{amiRNA-PHYB not functional because of target mRNA secondary structure?}

The construct ffgLUC ${ }^{\text {aimiRNA-PHYB }}$ showed correct splicing but this did not result in significant down regulation of PHYB mRNA levels in transformed Arabidopsis. Analysis of small RNA isolated from the plants expressing ffgLUC ${ }^{\text {aimiRNA-PHYB }}$ by stem-loop PCR [24] with specific primers did show that the expected aimiRNA ${ }^{\text {PHYB }}$ product is produced in these plants (Fig. 5e), but apparently it is not active against the PHYB mRNA. Also when the same amiR-PHYB was expressed directly from a $2 \times 35$ S-promoter, transformants did not show a PHYB silencing phenotype under constant $\mathrm{CR}$ (Additional file 1: Fig. S5). The lack of silencing by either aimiR-PHYB or amiR-PHYB suggests that the PHYB target sequence cannot be effectively silenced. For selection of the PHYB miRNA target sequence the WMD3-Web MicroRNA Designer online tool was used, which selects the best target sequence based on both target and off-target sequences [5]. However, recently it was shown that effectiveness of miRNA sequences also depends on the secondary structure of the target mRNA [36]. Indeed, when the secondary structure of the target mRNA is taken into account [37], it turns out that both the amiR-LUC and native miR319a target the mRNA at a part that is largely single stranded. In contrast, the chosen amiR-PHYB sequence targets the $P H Y B$ mRNA at an internal stem loop structure (Fig. 6). Possibly, this explains why the ffgLUC ${ }^{\text {aimiR-PHYB }}$ construct does not show effective silencing of $P H Y B$. 
In conclusion, the method with transgenes containing an amiRNA in their intron allows for combining ectopic overexpression of the transgene with silencing of a target gene of interest. Artificial miRNA genes containing functional clusters of miRNAs have been engineered [38]. Therefore, our concept of transgenes containing aimiRNA may be extended by multiple aimiRNAs in a single intron or in different introns in the same transgene provided that these aimiRNAs are still efficiently processed and allow dual/multiple functionality of the transgene.

\section{Additional file}

Additional file 1: Figure S1. Nucleotide sequences of all ff-gLUC/aimiRNA constructs used in this study. gatatc: - EcoR V site; gaattc: EcoR I site; capital letter: exon sequences; small letters black: intron sequences; small letters blue: Id-amiRNA sequences based on miR319a. Specific sequences targeting TCP, LUC or PHYB are underlined. 5'intron splice sequences are boxed (ag: 3'intron splice site, gt: 5'intron splice site). Figure S2. RT-PCR products sequence from ffgLUC $C_{\text {del }}$ aimiR-319a transgenic plant. Figure S3. A similar transformation efficiency were confirmed by quantify the DsRED gene expression in transient assay samples. The quantification data is normalized against the $\mathrm{N}$. benthamiana reference with UBI3 as internal control. Error bars represent standard error. Figure S4. Confirmation of mature aimiR-319a expression in stem-loop RT-PCR assay. Illustration of RT-PCR method for amplification of mature microRNA (A). Gel electrophoresis results from stem-loop RT (B). ffgLUC plant used as a positive control for endogenous mature miR319a. A mature microRNA specific forward primer and universal reverse primer were used for PCR amplification. Mature microRNA products were obtained using 25 cycling of RT-PCR and analysed on $4 \%$ agarose gel in 1 XTAE. Predicted products were compared with 50 bp DNA ladder. Figure S5. PHYB silencing phenotype under constant CR. Table S1. Stable transformation of Arabidopsis WT or Arabidopsis line ff-gLUC-1 with the different expression constructs. NA not applicable, *very low LUC activity; ${ }^{* *}$ low LUC activity. Table S2. List of primers were used in this work.

\section{Abbreviations}

imiRNA: Intronic microRNA ; aimiRNA: Artificial intronic microRNA; ffgLUC: Firefly genomic luciferase; TCP: TEOSINTE BRANCHED1/CYCLOIDEA/PROLIFERATING CELL FACTOR1; PHYB: PHYTOCHROME B; 5'ss: 5' splice sites.

\section{Authors' contributions}

US developed the method, carried out the experimental work and drafted the manuscript. MvH made Luminator system and analysed the data. MS developed the plant material. IA and HB helped writing the manuscript. SvdK supervised manuscript writing and the project. All authors read and approved the final manuscript.

\section{Author details}

' Laboratory of Plant Physiology, Wageningen University, Droevendaalsesteeg 1, 6708 PD Wageningen, The Netherlands. ${ }^{2}$ Center of Genomics and Bioinformatics, Academy of Sciences of Uzbekistan, University Street-2, Qibray Region, Tashkent, Uzbekistan 111215

\section{Acknowledgements}

The first author would like to thank the Erasmus Mundus Action 2 projects TIMUR.

\section{Competing interests}

The authors declare that they have no competing interests.

\section{Consent for publication}

All authors read and approved the final manuscript.
Ethics approvals and consent to participate Not applicable.

\section{Funding}

This research is funded by the STW Project (13149) 'Compact Plants'.

\section{Publisher's Note}

Springer Nature remains neutral with regard to jurisdictional claims in published maps and institutional affiliations.

Received: 26 April 2018 Accepted: 18 September 2018

Published online: 24 September 2018

\section{References}

1. Peng J, Richards DE, Hartley NM, Murphy GP, Devos KM, Flintham JE, Beales J, Fish $\sqcup$, Worland AJ, Pelica F, et al. 'Green revolution' genes encode mutant gibberellin response modulators. Nature. 1999:400:256-61.

2. Kasuga M, Liu Q, Miura S, Yamaguchi-Shinozaki K, Shinozaki K. Improving plant drought, salt, and freezing tolerance by gene transfer of a single stress-inducible transcription factor. Nat Biotechnol. 1999;17:287-91.

3. Chen $Y$, Han $Y$, Zhang M, Zhou S, Kong X, Wang W. Overexpression of the wheat expansin gene TaEXPA2 improved seed production and drought tolerance in transgenic tobacco plants. PLoS ONE. 2016;11:e0153494.

4. Karaba A, Dixit S, Greco R, Aharoni A, Trijatmiko KR, Marsch-Martinez N, Krishnan A, Nataraja KN, Udayakumar M, Pereira A. Improvement of water use efficiency in rice by expression of HARDY, an Arabidopsis drought and salt tolerance gene. Proc Natl Acad Sci U S A. 2007;104:15270-5.

5. Schwab R, Ossowski S, Riester M, Warthmann N, Weigel D. Highly specific gene silencing by artificial microRNAs in Arabidopsis. Plant Cell. 2006;18:1121-33.

6. ParkW, Zhai J, Lee JY. Highly efficient gene silencing using perfect complementary artificial miRNA targeting AP1 or heteromeric artificial miRNA targeting AP1 and CAL genes. Plant Cell Rep. 2009;28:469-80.

7. Watson E, MacNeil Lesley T, Arda HE, Zhu Lihua J, Walhout Albertha JM. Integration of metabolic and gene regulatory networks modulates the C. elegans dietary response. Cell. 2013;153:253-66.

8. Prelich G. Gene overexpression: uses, mechanisms, and interpretation. Genetics. 2012;190:841-54.

9. Bartel DP. MicroRNAs: genomics, biogenesis, mechanism, and function. Cell. 2004;116:281-97.

10. Reinhart BJ, Weinstein EG, Rhoades MW, Bartel B, Bartel DP. MicroRNAs in plants. Genes Dev. 2002;16:1616-26.

11. Yang GD, Yan K, Wu BJ, Wang YH, Gao YX, Zheng CC. Genomewide analysis of intronic microRNAs in rice and Arabidopsis. J Genet. 2012;91:313-24.

12. Yan K, Liu P, Wu CA, Yang GD, Xu R, Guo QH, Huang JG, Zheng CC. Stressinduced alternative splicing provides a mechanism for the regulation of microRNA processing in Arabidopsis thaliana. Mol Cell. 2012;48:521-31.

13. Lin SL, Miller JD, Ying SY. Intronic microRNA (miRNA). J Biomed Biotechnol. 2006;2006:26818.

14. Parsi S, Soltani BM, Hosseini E, Tousi SE, Mowla SJ. Experimental verification of a predicted intronic MicroRNA in human NGFR gene with a potential pro-apoptotic function. PLoS ONE. 2012;7:e35561.

15. Kashyap N, Pham B, Xie Z, Bleris L. Transcripts for combined synthetic microRNA and gene delivery. Mol BioSyst. 2013;9:1919-25.

16. Ruby JG, Jan CH, Bartel DP. Intronic microRNA precursors that bypass Drosha processing. Nature. 2007;448:83-6.

17. Knop K, Stepien A, Barciszewska-Pacak M, Taube M, Bielewicz D, Michalak M, Borst JW, Jarmolowski A, Szweykowska-Kulinska Z. Active 5' splice sites regulate the biogenesis efficiency of Arabidopsis microRNAs derived from intron-containing genes. Nucleic Acids Res. 2017:45:2757-75.

18. Ren G, Yu B. Post-transcriptional control of miRNA abundance in Arabidopsis. Plant Signal Behav. 2012;7:1443-6.

19. Nag A, King S, JackT. miR319a targeting of TCP4 is critical for petal growth and development in Arabidopsis. Proc Natl Acad Sci U S A. 2009;106:22534-9. 
20. Liang G, He H, Li Y, Yu D. A new strategy for construction of artificial miRNA vectors in Arabidopsis. Planta. 2012;235:1421-9.

21. Ali MA, Shah KH, Bohlmann H. pMAA-Red: a new pPZP-derived vector for fast visual screening of transgenic Arabidopsis plants at the seed stage. BMC Biotechnol. 2012;12:37.

22. Zhang X, Henriques R, Lin S-S, Niu Q-W, Chua N-H. Agrobacterium-mediated transformation of Arabidopsis thaliana using the floral dip method. Nat Protoc. 2006;1:641-6.

23. Wang B, Kashkooli AB, Sallets A, Ting H-M, Ruijter NCA, Olofsson L, Brodelius P, Pottier M, Boutry M, Bouwmeester H, van der Krol AR. Transient production of artemisinin in Nicotiana benthamiana is boosted by a specific lipid transfer protein from A. annua. Metab Eng. 2016;38:159-69.

24. Varkonyi-Gasic E, Wu R, Wood M, Walton EF, Hellens RP. Protocol: a highly sensitive RT-PCR method for detection and quantification of microRNAs. Plant Methods. 2007;3:12.

25. Luke Mankin S, Allen GC, Thompson WF. Introduction of a plant intron into the luciferase gene ofPhotinus pyralis. Plant Mol Biol Report. 1997;15:186-96.

26. Palatnik JF, Wollmann H, Schommer C, Schwab R, Boisbouvier J, Rodriguez R, Warthmann N, Allen E, Dezulian T, Huson D, et al. Sequence and expression differences underlie functional specialization of Arabidopsis microRNAs miR159 and miR319. Dev Cell. 2007;13:115-25.

27. Alvarez JP, Furumizu C, Efroni I, Eshed Y, Bowman JL. Active suppression of a leaf meristem orchestrates determinate leaf growth. Elife. 2016;5:e15023.

28. Berezikov E, Chung W-J, Willis J, Cuppen E, Lai EC. Mammalian mirtron genes. Mol Cell. 2007;28:328-36.
29. Sibley CR, Seow Y, Saayman S, Dijkstra KK, El Andaloussi S, Weinberg MS, Wood MJA. The biogenesis and characterization of mammalian microRNAs of mirtron origin. Nucleic Acids Res. 2012;40:438-48.

30. Tong YA, Peng H, Zhan C, Fan L, Ai T, Wang S. Genome-wide analysis reveals diversity of rice intronic miRNAs in sequence structure, biogenesis and function. PLoS ONE. 2013;8:e63938.

31. Meng Y, Shao C. Large-scale identification of mirtrons in Arabidopsis and rice. PLOS ONE. 2012;7:e31163.

32. Ha M, Kim VN. Regulation of microRNA biogenesis. Nat Rev Mol Cell Biol. 2014;15:509-24.

33. Naqvi AR, Sarwat M, Hasan S, Roychodhury N. Biogenesis, functions and fate of plant microRNAs. J Cell Physiol. 2012;227:3163-8.

34. Schuler MA. Splice site requirements and switches in plants. In: Reddy ASN, editor. Nuclear pre-mRNA processing in plants. Berlin: Springer; 2008. p. 39-59.

35. Wang BB, Brendel V. Genomewide comparative analysis of alternative splicing in plants. Proc Natl Acad Sci U S A. 2006;103:7175-80.

36. Zheng Z, Reichel M, Deveson I, Wong G, Li J, Millar AA. Target RNA secondary structure is a major determinant of miR159 efficacy. Plant Physiol. 2017:174:1764

37. Buratti E, Baralle FE. Influence of RNA secondary structure on the PremRNA splicing process. Mol Cell Biol. 2004;24:10505-14.

38. Wang T, Xie Y, Tan A, Li S, Xie Z. Construction and characterization of a synthetic MicroRNA cluster for multiplex RNA interference in mammalian cells. ACS Synth Biol. 2016;5:1193-200.
Ready to submit your research? Choose BMC and benefit from:

- fast, convenient online submission

- thorough peer review by experienced researchers in your field

- rapid publication on acceptance

- support for research data, including large and complex data types

- gold Open Access which fosters wider collaboration and increased citations

- maximum visibility for your research: over 100M website views per year

At BMC, research is always in progress.

Learn more biomedcentral.com/submissions 